Techniques \& Culture

\title{
Conserver pour rester terriens
}

Alain Maillet

\section{(2) OpenEdition}

Journals

Édition électronique

URL : https://journals.openedition.org/tc/8821

DOI : $10.4000 /$ tc. 8821

ISSN : 1952-420X

\section{Éditeur}

Éditions de l'EHESS

\section{Édition imprimée}

Date de publication : 17 mai 2018

Pagination : 184-201

ISBN : 978-2-7132-2750-9

ISSN : 0248-6016

Référence électronique

Alain Maillet, « Conserver pour rester terriens », Techniques \& Culture [En ligne], 69 | 2018, mis en ligne le 17 mai 2020, consulté le 29 septembre 2022. URL : http://journals.openedition.org/tc/8821 ; DOI : https://doi.org/10.4000/tc.8821 


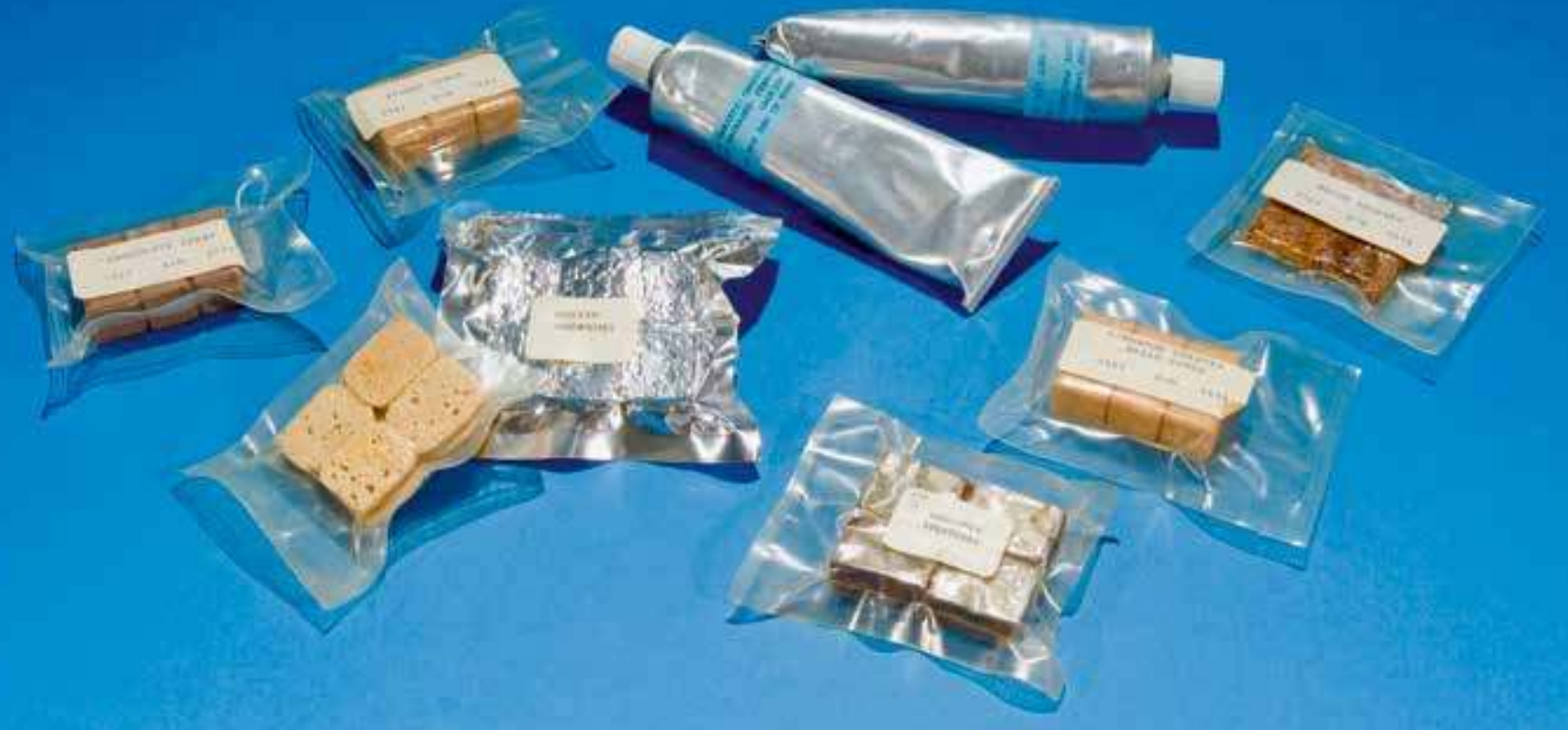




\section{Alain Maillet \\ Conserver pour rester terriens}

Dès les premiers vols spatiaux, l'alimentation des cosmonautes ou astronautes s'est avérée un élément important dans les programmes de vol. Son importance s'est accrue au fil des missions, à mesure que leur durée augmentait. Elle est un véritable challenge des missions actuelles et futures dont on distingue 3 principaux facteurs clés: la santé et les performances de l'astronaute, l'aspect psychologique et la contrainte logistique et financière.

La consommation alimentaire dans l'espace a beaucoup évolué entre les premières missions et celles d'aujourd'hui. Elle évoluera encore pour s'adapter aux contraintes et enjeux des missions futures. Yuri Gagarine a été le premier à expérimenter la nourriture spatiale. Il consommait alors des aliments en tubes; il disposait de 9 produits (incluant soupes et jus) pendant son vol de 108 minutes en 1961 (Shramchenko 2015). Lors des vols américains habités Mercury (1961-1963), la NASA proposa aussi de la nourriture en tube à ses astronautes accompagnée d'aliments déshydratés qui permettaient à la fois de diversifier les repas tout en restant léger. L'allongement progressif de la durée des vols habités n'a fait que renforcer l'importance de l'alimentation pour les aspects «support vie ${ }^{1} »$ et le maintien en bonne santé des astronautes: les efforts de nombreuses équipes regroupant techniciens et ingénieurs, nutritionnistes et médecins mais aussi des microbiologistes ont permis d'utiliser de nouvelles solutions de production et de conservation, augmentant ainsi la variété et le nombre de recettes disponibles (Ahmed 1988, Bourland 1993, Lane 2016). La fourniture de repas de qualité aux astronautes, tant d'un point de vue gustatif que du point de vue de sa valeur nutritionnelle (équilibre des taux de macronutriments - protéines, lipides, glucides - et de micronutriments - vitamines, minéraux...), est primordiale pour que les équipages gardent un état de forme et de santé optimal pendant toute la durée de leur mission. Elle est aussi importante d'un point de vue psychologique: comme ça l'est sur Terre mais, peutêtre plus encore, la valeur «plaisir » de manger doit être préservée, car les repas sont un des seuls moments où les équipages sont ensemble pour un instant d'échange et de convivialité. 
L'alimentation en tube a été privilégiée au début des vols orbitaux par crainte d'une mauvaise ingestion et digestion des aliments en micropesanteur. Une fois ces craintes dissipées, les tubes et les cubes alimentaires furent rapidement abandonnés, car ils présentaient plus de désavantages que de points positifs: le goût et la texture des produits étaient bien différents des recettes attendues (Bourland 1993, Perchonok 2002), ainsi de nombreux produits étaient ramenés au sol non consommés. Des premiers vols spatiaux soviétiques ou américains, seuls subsistent aujourd'hui les aliments ou boissons déshydratés qui fournissent notamment aux équipages des boissons chaudes ou froides mais aussi des soupes et bien d'autres recettes (par exemple: de la soupe aux champignons), et les aliments à faible teneur en eau, que l'on retrouve aujourd'hui également sous forme de biscuits ou barres vitaminées. Les possibilités de chauffer les aliments ou d'avoir des boissons réhydratées chaudes sont apparues à partir des vols Apollo (Perchonok 2002) pour les Américains et du vol Soyuz 9 en 1970 pour les Soviétiques (Ahmed 1988).

Une prise alimentaire inadaptée a un effet négatif sur la santé des équipages (Lane 2014, Smith 2014): elle peut ainsi augmenter les modifications engendrées par la micropesanteur (par exemple la baisse de la densité osseuse, la perte de masse et force musculaire...). Aussi, les équipes médicales portent une attention particulière au suivi nutritionnel et à l'alimentation des équipages. Rapidement après les premiers vols, les apports journaliers nutritionnels pour les astronautes se sont avérés similaires à ceux recommandés au sol par l'Organisation mondiale de la santé (auxquels est appliqué un facteur modérateur pour prendre en compte la baisse d'activité), soit de l'ordre de 2200-2500 kcal/jour pour un homme (Perchonok 2002, Lane 2014), et adaptés au métabolisme de base de chaque individu (masse corporelle, bilan métabolique avant le vol). En effet, bien que les astronautes pratiquent de façon régulière l'exercice physique (tapis roulant, vélo ergomètre ou banc de musculation) à bord de la Station spatiale internationale (deux heures et demie par jour), leurs muscles (notamment au niveau du dos et des membres inférieurs) ne sont pas du tout sollicités pendant le reste du temps. Avec l'augmentation progressive de la durée des vols de quelques heures (Vostok, Mercury) à plusieurs jours (Soyuz, Gemini, Apollo, et la navette américaine ou Shuttle) puis plusieurs mois avec les stations orbitales (Salyut et Mir pour les Russes, Skylab pour les Américains; les vols Shuttle-Mir, programme précurseur de l'actuelle Station spatiale internationale), les aliments et recettes disponibles pour les équipages n'ont fait que se diversifier. Aujourd'hui plusieurs types d'aliments sont disponibles:

- Les boissons et aliments déshydratés ou maintenant lyophilisés (du bœuf Strogonoff, des œufs brouillés, des spaghettis sauce bolognaise, un cocktail de crevettes) qui sont réhydratés en vol avec de l'eau froide (jus de fruits) ou chaude (soupes, café, thé...) à partir de l'un des deux distributeurs d'eau de la station. Les poches utilisées ont un volume maximum de $250 \mathrm{ml}$.

- Les aliments à faible teneur en eau (principalement des fruits secs ou des biscuits).

- Les aliments frais (uniquement disponibles pendant une courte période après l'arrivée des vaisseaux ravitailleurs: fruits ou légumes ou, par exemple, des tortillas qui peuvent remplacer l'assiette pour composer un plat) ou les aliments non modifiés (barres de céréales, cacahuètes...). 
- Les aliments en conserve ou thermostabilisés qui sont disponibles soit sous forme de boîtes notamment fournies par la Russie, la France ou l'Europe (voir infra) et sont réchauffés dans le four de la partie russe de la station (système de mâchoires métalliques qui se placent sur les côtés des boîtes et chauffent les boîtes à $65^{\circ} \mathrm{C}$ par séquence de quinze minutes) situé à l'extrémité de la table où l'équipage peut préparer les repas, soit sous forme de sachets flexibles opaques fournis par la NASA, qui sont chauffés dans des mallettes qui bloquent les poches entre deux parois chauffantes. Par exemple: morceaux de bœuf avec des champignons, porc grillé, salade de fruits tropicaux...

- Les aliments stérilisés par radiations ionisantes qui sont fournis par la NASA en poches souples et opaques. Par exemple des saucisses pour le petit-déjeuner, de la dinde fumée...

Au total, ce sont près de 350 recettes qui sont aujourd'hui disponibles pour les équipages de la Station spatiale internationale; la production de ces recettes est majoritairement réalisée par les Américains et les Russes (chaque agence fournie 50 \% de la masse de nourriture envoyée régulièrement vers la station). Chaque astronaute va participer avant son vol à des séances de dégustation des différentes recettes disponibles et établir sa liste de préférence afin que les menus constitués par les équipes au sol ne comprennent pas des aliments ou recettes que l'astronaute n'aime pas. Une fois à bord, les astronautes disposent de conteneurs qui renferment à la fois la nourriture russe et américaine: ces conteneurs sont accompagnés de recommandations adaptées à chaque astronaute pour une période de 15 jours, celui-ci choisit donc au final ses menus journaliers. Les astronautes disposent aussi de nombreuses possibilités d'assaisonnement, et ils n'hésitent pas à les utiliser pour renforcer les goûts, ce qui peut parfois donner des associations originales.

En plus de ces deux producteurs principaux, il faut rajouter d'autres acteurs: le Centre national d'études spatiales (CNES) fournit régulièrement des repas pouvant être utilisés par tous les équipages soit pour des repas évènementiels (anniversaires, fêtes, relève d'équipage) avec une fréquence d'un ou deux repas par mois (35 recettes disponibles), soit dans le cadre de protocoles scientifiques et notamment d'expérience de nutrition: c'est le cas de l'expérience française ENERGY, sélectionnée par l'Agence spatiale européenne (European Space Agency ESA), pendant laquelle l'astronaute a un régime imposé de quatre repas consécutifs sur une période de 36 heures. L'objectif de cette expérience est de déterminer la dépense énergétique des astronautes au cours d'un vol de longue durée (supérieur à trois mois). D’autres agences (l'ESA et l'agence japonaise) fournissent des repas lorsque l'un de leurs astronautes est présent à bord de la station. Ces recettes sont disponibles dans des conteneurs «bonus» qui comportent les choix personnels de l'astronaute composés de produits commerciaux vérifiés par la NASA au niveau microbiologique. En plus des conteneurs «bonus», les astronautes peuvent aussi recevoir des aliments choisis par leur famille; ces aliments étant également vérifiés par la NASA avant l'envoi. 
Kit de nourriture tel qu'utilisé par les astronautes des vols Mercury

Plusieurs éléments sont déshydratés et nécessitent de l'eau avant ingestion, d'autres sont prêts à l'usage: soupe aux champignons, jus orange pamplemousse, boisson à la noix de coco, jus d'ananas, poulet en sauce, poires, fraises. Sur chaque emballage, on peut identifier la nature du contenu, la quantité d'eau nécessaire pour réhydrater le tout ou si l'aliment peut être consommé tel quel.

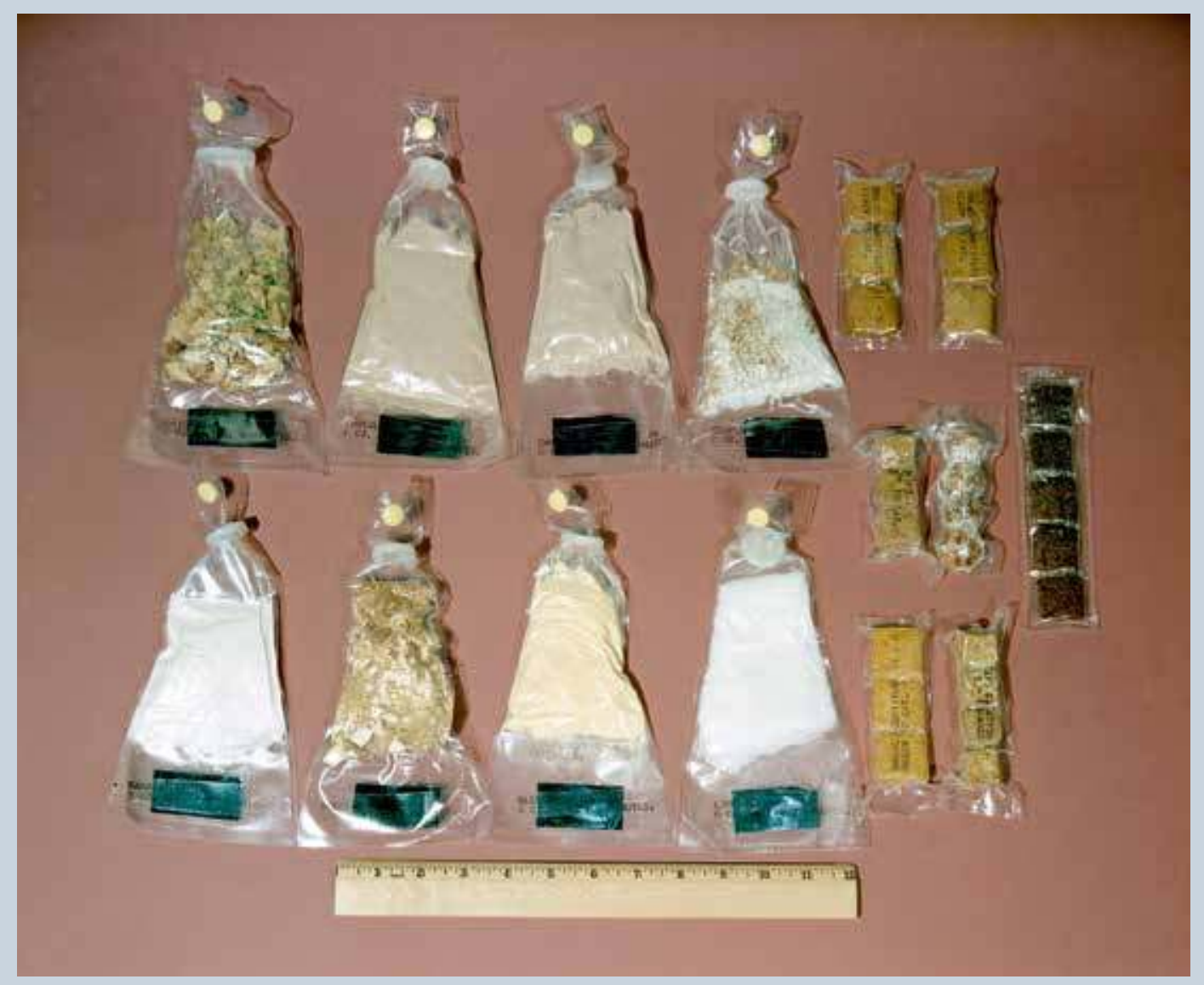




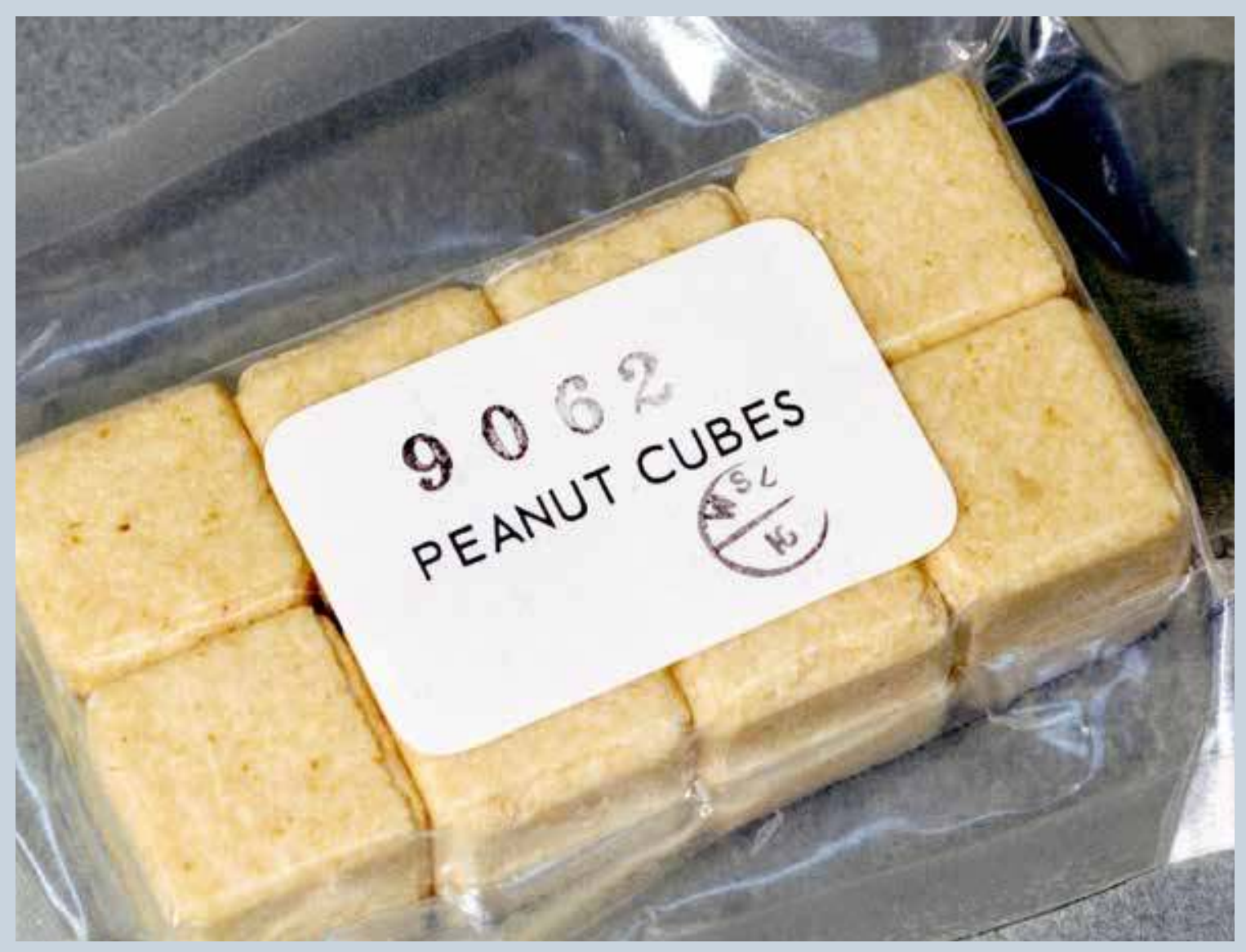

2. Éléments pour l'alimentation des astronautes pendant les missions américaines Mercury et Gemini: exemple de cubes alimentaires, utilisés comme équivalents de biscuits. 


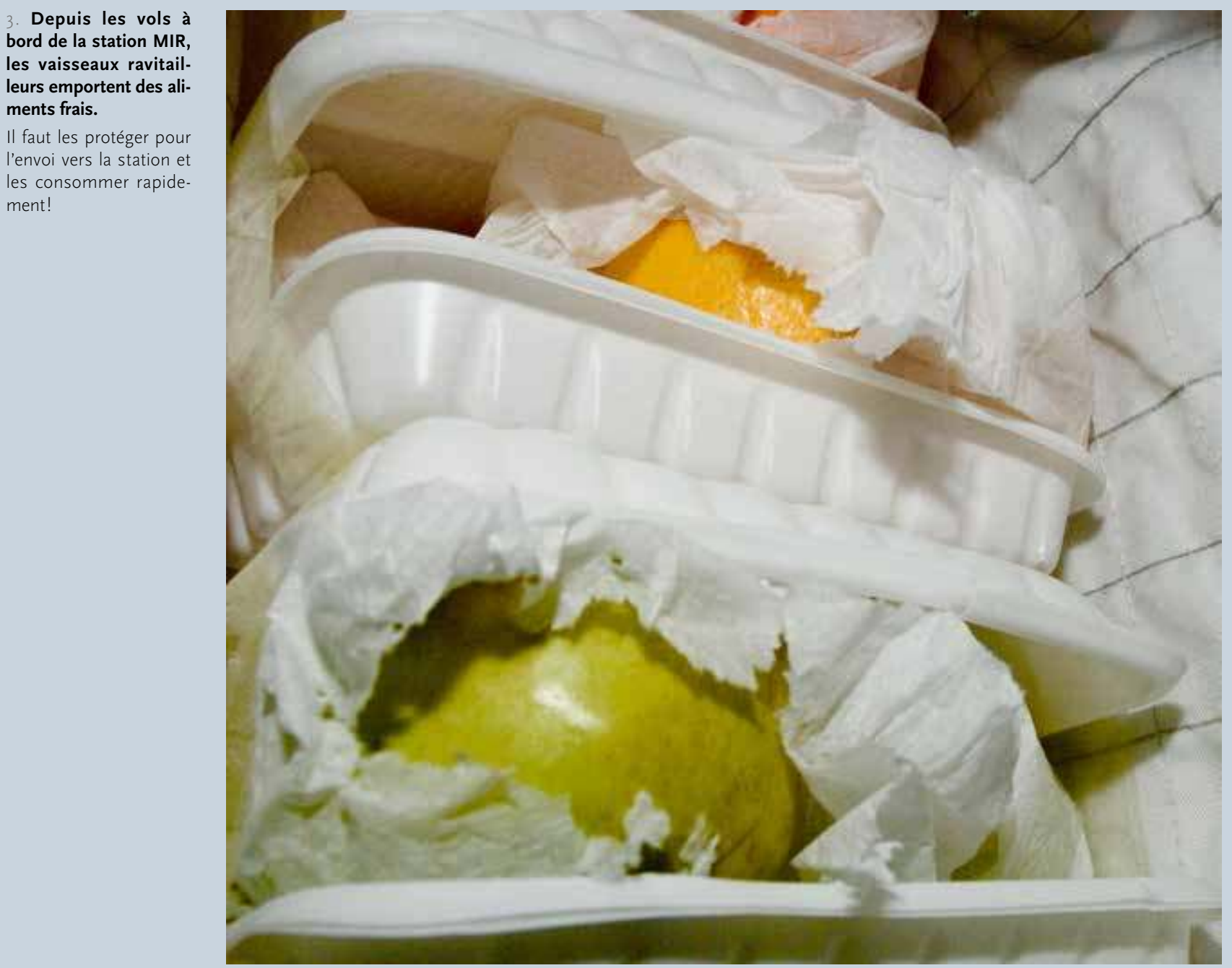


4. Fruits et Légumes frais sont disponibles pendant une courte période pour les équipages de la Station spatiale internationale après l'arrivée des vais. seaux ravitailleurs.

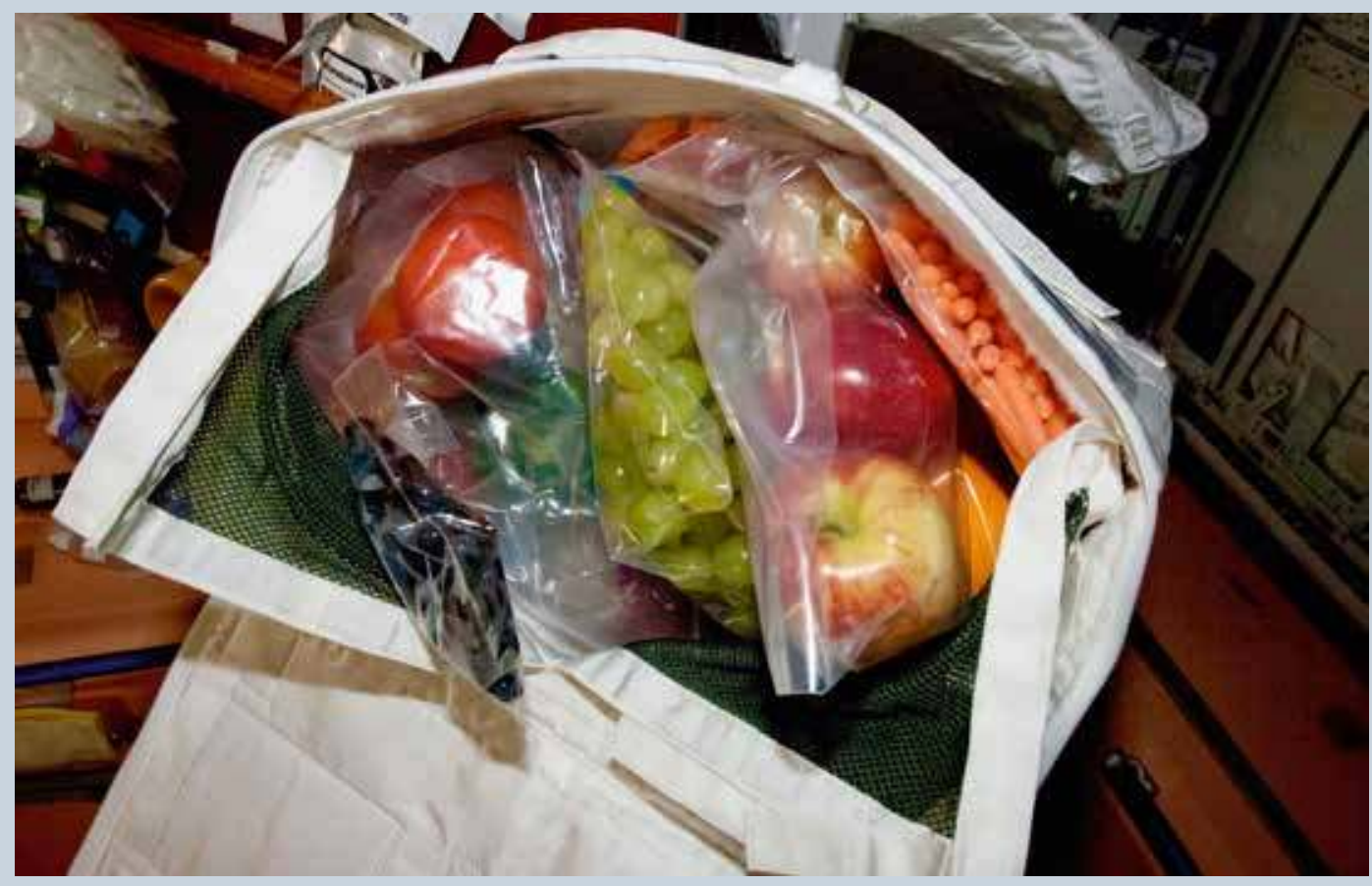


5. En plus de la grande variété de recettes disponibles à bord de la station, les possibilités d'assaisonnements sont nombreuses!

Saumon au citron sur tortilla assaisonnée avec de la sauce piquante et de la crème à l'ail. La tortilla servant d'as. siette.

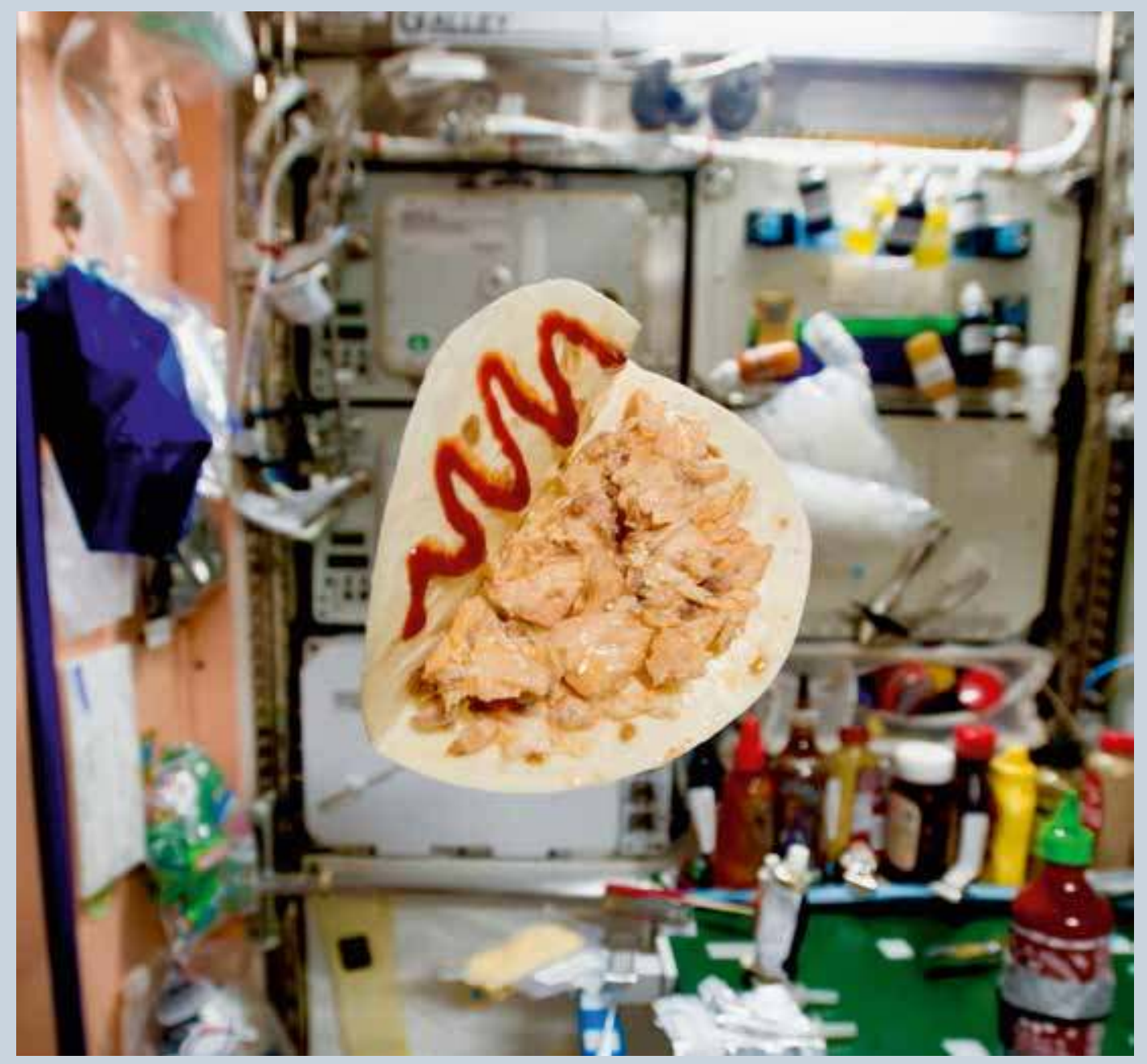


6. Les repas évènementiels fournis par le CNES permettent également la réalisation d'expériences de nutrition telles que celle de l'ESA, ENERGY, qui vise à déterminer la dépense énergétique des astronautes pendant des vols de longue durée (supérieure à trois mois).

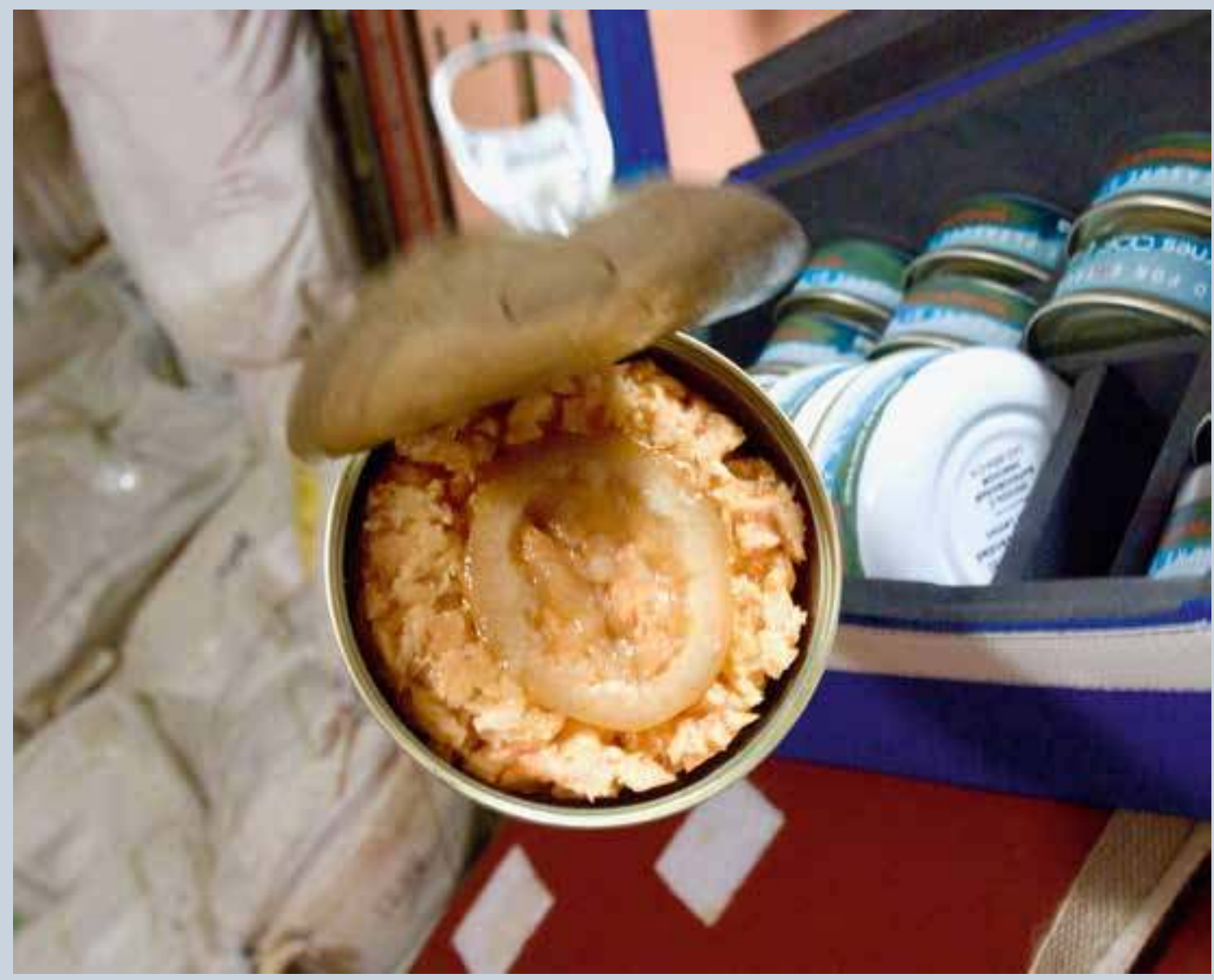


7. Chaque astronaute peut choisir son menu chaque jour; il dispose d'une liste de recommandations pour une période de deux semaines.

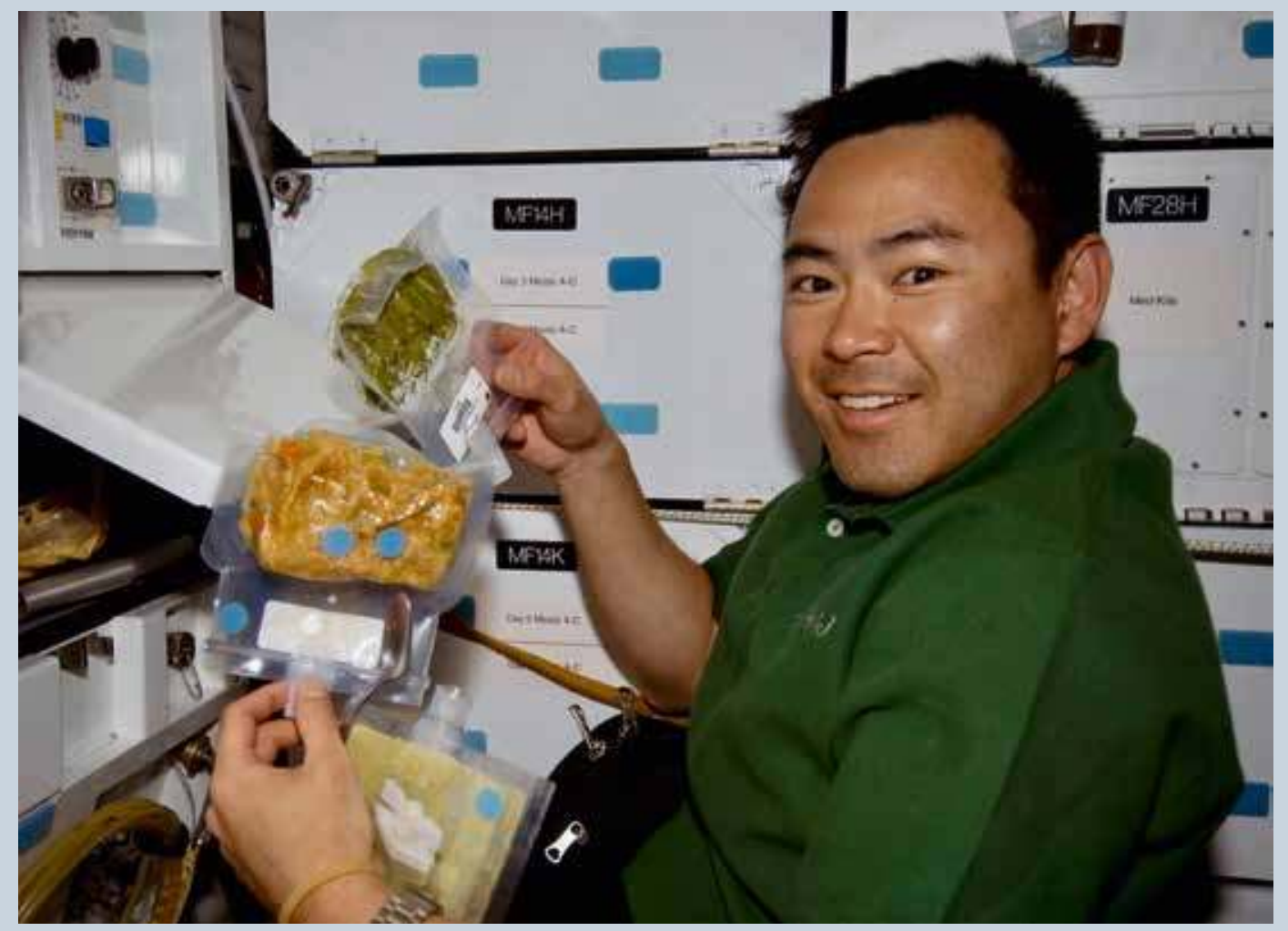


8. Des expériences scientifiques étu dient la culture de salades dans la Station spatiale internationale afin de préparer les vols futurs de plus longue durée. Un avantage, les astronautes peuvent «goûter» le produit de leur travail!

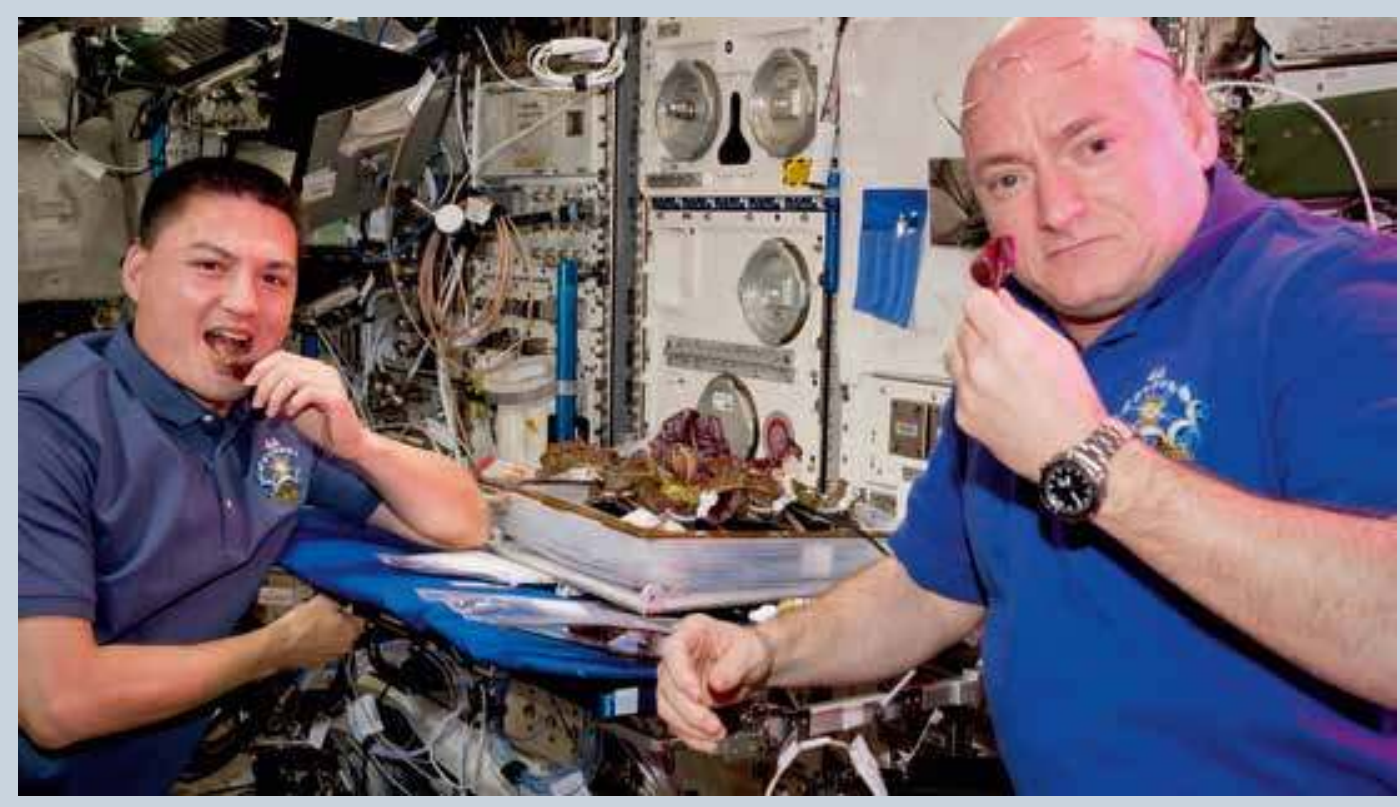




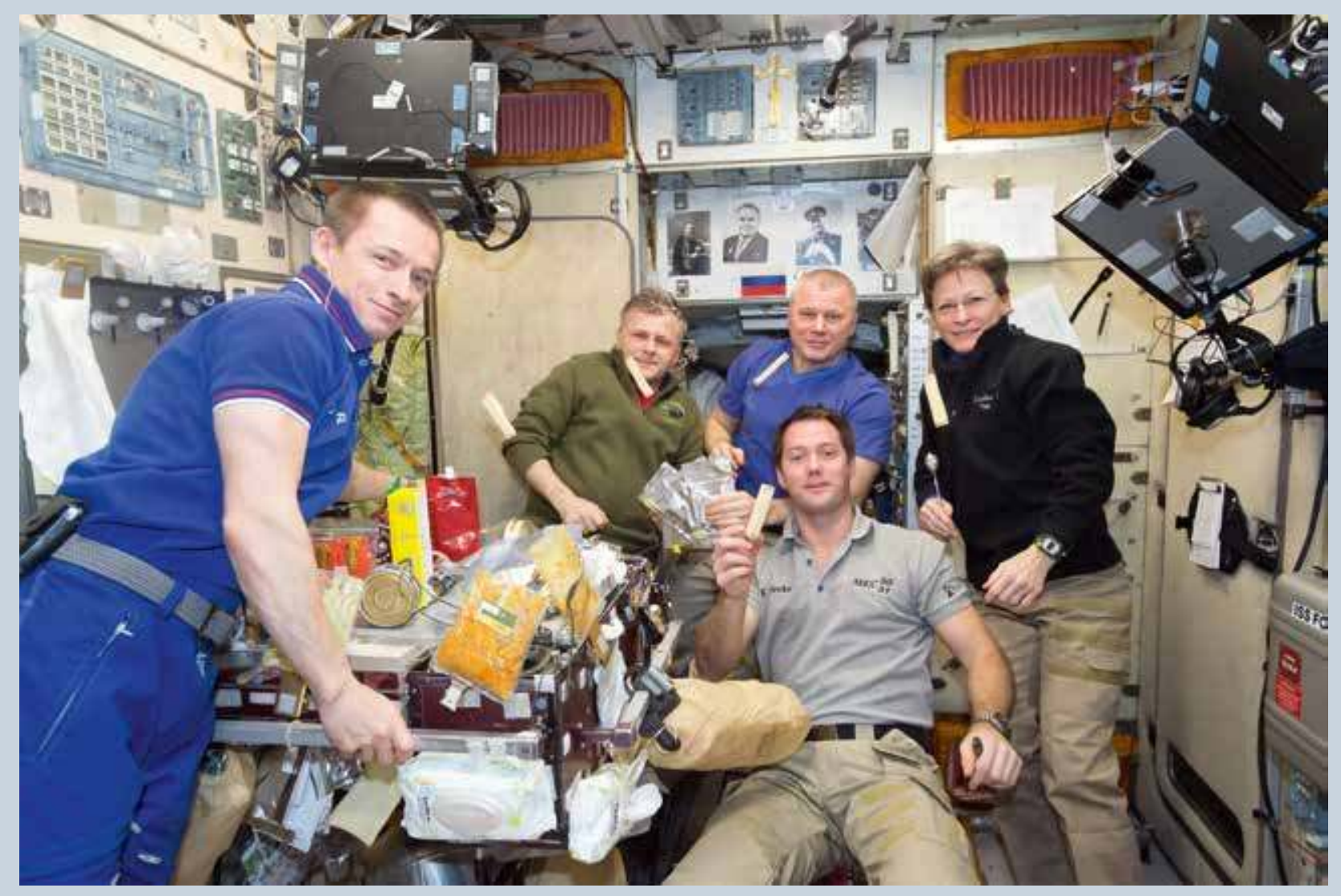

9. Les astronautes disposent de «bonus» containers par lesquels leurs familles peuvent leur faire envoyer des aliments qui ont une valeur «personnelle». Illustration: dégustation de fromage français durant la mission Proxima.

10. Fromage ou dessert (ci-contre, des macarons)! 


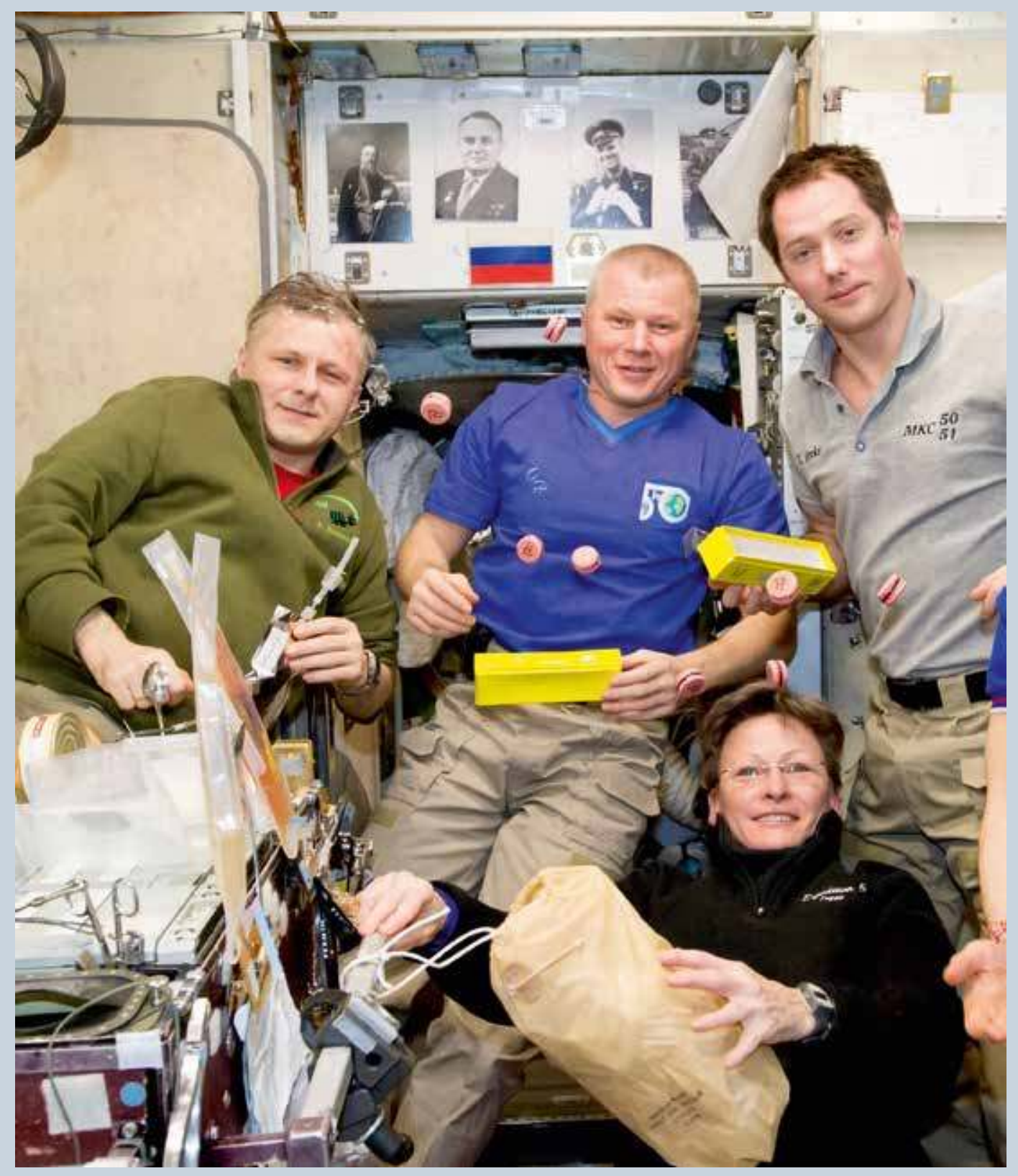


L'alimentation dans l'espace est donc très importante pour le bon déroulement des missions. Elle participe au maintien des performances physiques des équipages. Comme nous l'avons décrit précédemment, tout est fait au niveau du régime alimentaire proposé pour préserver l'équilibre énergétique (sur l'ensemble de la durée de la mission mais aussi lors d'évènements particuliers tels que les sorties extra-véhiculaires qui imposent de par leur durée et l'effort induit une augmentation importante de la dépense énergétique). Elle participe aussi à la préservation de tous les systèmes de l'organisme (muscle, os, cardio-vasculaire...) pour lutter contre les effets de la micropesanteur (Lane 2013). Enfin, l'alimentation (grâce aux repas) joue un rôle très important au niveau social pour l'équilibre des équipages: c'est un moment privilégié de la vie dans les stations spatiales (Sipes 2016). Tout au long de la journée, chaque astronaute réalise les différentes tâches de son planning (expériences scientifiques, maintenance de la station, entraînement physique, activités liées au suivi médical...), mais toutes ces activités sont réparties dans les différents modules de la station spatiale et il est rare qu'elles impliquent plusieurs astronautes en même temps. Aussi les repas (et notamment ceux du soir ou du dimanche) sont un moment privilégié de convivialité: ils sont les seuls moments où les équipages peuvent partager ensemble leur culture et leurs habitudes, et ainsi avoir des temps d'échange, rythmant le temps social mais également physiologique. Ces aspects seront encore renforcés avec l'augmentation de la durée des vols futurs d'exploration (pour une mission vers Mars, les scenarii envisagés sont de l'ordre de trois ans!).

Dans cette perspective, d'autres aspects liés à l'alimentation vont entrer en jeu. La quantité importante de nourriture nécessaire pour de telles missions (durée et nombre d'astronautes) est incompatible avec la taille actuelle des lanceurs, il faudra donc positionner de la nourriture avant l'arrivée de l'équipage ou de façon périodique pendant le séjour dans la base (Lane 2016). Cet aspect logistique, et donc financier (une erreur importante sur la quantité embarquée serait critique pour la santé de l'équipage et pour le coût de la mission), sera nécessairement à prendre en compte pour des vols de très longue durée, comme vers Mars par exemple (Douglas 2016). De plus, la conservation des aliments et la préservation de leur qualité nutritive doivent faire l'objet d'améliorations significatives (Zwart 2009, Perchonok 2012) : aujourd'hui, les aliments produits pour la Station spatiale internationale sont considérés comme stables pour une durée de trois ans (Catauro 2012, Cooper 2017), cela ne sera pas suffisant pour ces nouvelles missions, il faudra fournir des garanties supérieures (la cible visée aujourd'hui est de cinq ans). C'est un véritable challenge, notamment pour la conservation des vitamines avec les techniques de production et de conservation actuelle. La production d'aliments in situ (dans le vaisseau ou dans la base) permettra pour une petite partie d'optimiser la masse de nourriture à emporter pour la mission, mais aussi la fourniture de quelques aliments frais; des expériences récentes réalisées à bord de la Station spatiale internationale ont montré que la culture de salades était tout à fait possible et qu'elle permettait aussi d'avoir de nouveaux aliments frais à bord pouvant être consommés. Depuis plusieurs années, des travaux ont permis d'identifier quels aliments pourraient être cultivés en boucle fermée et de façon autonome (entre autres: la salade, les tomates, le riz, la pomme de terre, le soja, les oignons...). Enfin, l'« agriculture spatiale» (Lasseur 
2010, Wheeler 2017) participera au recyclage de l'air et de l'eau et fournira des aliments aux astronautes. Ces nouveaux procédés de production devront être évalués en termes de sécurité, de santé et d'acceptabilité par les équipages; l'autre intérêt ou défi de ces innovations résidera dans une utilisation «terrestre» dans des zones de culture difficiles (soit du fait du climat ou de faibles conditions de ressources).

\section{Notes}

1. Le «support vie» est un terme générique qui couvre tout ce qui permet à l'être humain de vivre dans un milieu extrême-comme l'espace. Il recouvre

\section{Iconographie}

Image d'ouverture. Éléments pour alimentation des astronautes pendant les missions américaines Mercury et Gemini. Les tubes sont présents (ainsi que les éléments déshydratés non représentés sur la photo); apparition

des activités dans de nombreux domaines: la nourriture, l'eau, l'air respirable, la contamination, la gestion des déchets, etc.

\section{L'auteur}

Alain Maillet, docteur ès sciences de l'université Claude Bernard à Lyon, travaille à Medes (Institut de Médecine et de Physiologie Spatiales à Toulouse), a rejoint, en septembre 2001, le Cadmos, service du Centre national d'études spatiales à Toulouse, qui met en place le support nécessaire aux équipes scientifiques françaises et internationales pour la réalisation de leurs protocoles expérimentaux à bord de la Station spatiale internationale. Il compte en 2018 cinq pôles d'activités: sciences de la matière, physique fondamentale, biologie \& exobiologie, exploitation, et le pôle sciences de la vie. Ce dernier regroupe dix personnes de Medes sous la responsabilité d'A. Maillet.

\section{Références}

Ahmed, S. 2017 «Comparison of Soviet and U.S. space food and nutrition programs », [En Ligne] : ntrs.nasa. gov/archive/nasa/casi.ntrs.nasa.gov/19890010688.pdf.

Bourland, C.T. 1993 «The development of food systems for space», Trends in Food Science \& Technology 4 : 271-276.

Catauro, P.M. \& M.H. Perchonok 2012 «Assessment of the long-term stability of retort pouch foods to support extended duration spaceflight », Journal of Food Science 71 (1): S29-S39.

Cooper, M., Perchonok, M. \& G. L. Douglas 2017 «Initial assessment of the nutritional quality of the space food system over three years of ambient storage», npj Microgravity 3, 17. doi: 10.1038/s41526-017-0022-z.
Douglas, G.L. et al. 2016 «Evidence report: Risk of performance decrement and crew illness due to an inadequate food system ", National Aeronautics and Space Administration, Houston TX: 1-48.

Lane, H.W. et al. 2013 «The role of nutritional research in the success of human space flight », Advances in Nutrition 4: 521-523.

Lane, H.W., Zwart, S.R., Kloeris, V.L. \& S. M. Smith 2014 «Food and nutrition for space flight», in C.D. Berdanier, J.T. Dwyer \& D. Heber dir. Handbook of Nutrition and Food. Boca Raton: CRC Press ( $3^{e}$ éd.): 381-401.

Lane, H.W., Smith, S.M. \& V. L. Kloeris 2016 « Metabolism and Nutrition », in A.E. Nicogossian et al. dir., Space 
Physiology and Medicine. From Evidence to Practice. New York: Springer (4e édo .) : 307-321.

Lasseur, C. et al. 2010 «MELiSSA: The European Project of Closed Life Support System », Gravitational and Space Biology 23 (2) : 3-12.

Perchonok, M.H. \& C.T. Bourland 2002 «NASA food systems: Past, present, and future», Nutrition 18 : 913-920.

Perchonok, M.H., Cooper, M.R. \& P. M. Catauro 2012 «Mission to Mars: Food production and processing for the final frontier», Annual Review of Food Science and Technology 3 : 311-330.

Shramchenko, T. 2015 «Cosmic crab, star salmon and space sturgeon: What's on a cosmonaut's menu?». [En Ligne] : rbth.com/science_and_tech/2015/04/11/ cosmic_crab_star_salmon_and_space_sturgeon_ whats_on_a_cosmon_45141.html.
Sipes, W.E., Polk, J.D., Beven, G. \& M. Shepanek 2016 «Behavioral health and performance», in A.E. Nicogossian et al. dir. Space Physiology and Medicine: From Evidence to Practice. New York: Springer (4e éd.) : 367-389.

Smith, S.M. et al. 2014 « Men and women in space: Bone loss and kidney stone risk after long-duration spaceflight », Journal of Bone and Mineral Research 29 (7): 1639-1645.

Zwart, S.R. et al. 2009 « Assessment of nutrient stability in foods from the space food system after long-duration spaceflight on the ISS », Journal of Food Science 74 (7): H209-H217.

Wheeler, R.M. 2017 «Agriculture for space: People and places paving the way», Open Agriculture 2: 14-32.

\section{Pour citer l'article}

Maillet, A. 2018 «Conserver pour rester terriens», Techniques\& Culture 69 «Le temps des aliments », p. 184-201. 
